\title{
Correlations, Spectra, and Instability of Phase-Space Density Fluctuations in Open-Cluster Models
}

\author{
V. M. Danilov* and S. I. Putkov** \\ Kourovka Astronomical Observatory, Ural Federal University, Ekaterinburg, 620000 Russia
}

Received December 28, 2012; in final form, February 15, 2013

\begin{abstract}
The dynamical evolution of six open star cluster models is analyzed using the correlation and spectral analysis of phase-space density fluctuations. The two-time and mutual correlation functions are computed for the fluctuations of the phase-space density of cluster models. The data for two-time and two-particle correlations are used to determine the correlation time for phase-space density fluctuations $\left((0.1-1) \tau_{\text {v.r. }}\right.$, where $\tau_{\text {v.r. }}$ is the violent relaxation time of the model $)$ and the average phase velocities of the propagation of such fluctuations in cluster models. These velocities are $2-20$ times smaller than the root mean square velocities of the stars in the cluster core. The power spectra and dispersion curves of phase-space density fluctuations are computed using the Fourier transform of mutual correlation functions. The results confirm the presence of known unstable phase-space density fluctuations due to homologous fluctuations of the cluster cores. The models are found to exhibit a number of new unstable phase-space density fluctuations (up to 32-41 pairs of fluctuations with different complex conjugate frequencies in each model; the e-folding time of the amplitude growth of such fluctuations is $(0.4-10) \tau_{\text {v.r. }}$ and their phases are distributed rather uniformly). Astrophysical applications of the obtained results (irregular structure of open star clusters, formation and decay of quasi-stationary states in such clusters) are discussed.
\end{abstract}

DOI: $10.1134 / \mathrm{S} 199034131302003 \mathrm{X}$

Keywords: stars: kinematics and dynamics-Galaxy: open clusters and associations: general

\section{INTRODUCTION}

Recently, Chavanis [1-3] derived theoretical estimates for the fluctuations of phase-space density (PSD) and the corresponding correlation functions for spatially uniform and nonuniform systems with long-range interactions (including self-gravitating systems). To this end, the above author used kinetic equations written with a number of simplifying assumptions. The formulas for the correlation functions derived by Chavanis [1-3] have a rather complex form making them difficult to use for analyzing dynamic processes in such systems. In our opinion, it is more productive to compute the correlation functions directly by numerically integrating the equations of motion of gravitating particles in the problems of clustering of galaxies and the evolution of the Universe (see, e.g., [4-6]), and also when modeling the dynamics of open star clusters $(\mathrm{OCl})[7]$.

In one of our previous papers [7] we computed the two-time correlation functions for $r=|\mathbf{r}|, v=|\mathbf{v}|$, and the energy $\varepsilon$ per unit star mass, as well as twoparticle correlations of $r, v, \varepsilon$, stellar number density $n=n(\mathbf{r}, t)$ and PSD $f=f(\mathbf{r}, \mathbf{v}, t)$ for open star

\footnotetext{
*E-mail: Vladimir.Danilov@usu.ru

${ }^{* *}$ E-mail: Putkov_S@mail.ru
}

cluster models in the vicinity of these stars. Here $\mathbf{r}$ and $\mathbf{v}$ are the star's radius vector and velocity vector, respectively. We used the correlation data to determine the correlation times $\tau_{c}$ and radii $r_{c}$ in the above parameter spaces and estimate the parameters of the density, potential, and PSD waves in the star cluster models. We also found evidence of the formation of polarized clouds when interstellar distances reach a certain value, and revealed the dominating flow of PSD correlations from the region of strong correlations to the region of weak correlations. Such a flow results in the flow of kinetic energy $T$ towards the center of the cluster (from the region of largescale to the region of small-scale PSD fluctuations (see [8]) with lower amplitudes and higher frequencies). According to Danilov and Putkov [7], the rates of heating of cluster models by this flow are equal to $\dot{T} \sim(0.1-1.6) T / \tau_{\text {v.r. }}$, where $T$ is the total kinetic energy of stars with clustercentric distances $r \leq 0.5 R_{t}$; $\tau_{\text {v.r. }}$ is the initial time scale of violent relaxation of the cluster model, which we set equal to $\tau_{\text {v.r. }} \simeq 2.6 \bar{t}_{\text {cr }}$ in accordance with the estimates of Aarseth [9]; $\bar{t}_{\mathrm{cr}}$ is the average initial cluster crossing time, and $R_{t}$ is the tidal radius of the cluster [10]. We determined $\tau_{\text {v.r. }}$ and $t_{\mathrm{cr}}$ from the initial parameters of $\mathrm{OCl} \bmod -$ els. Danilov and Putkov [7] found evidence of weak 
turbulence in stellar motions in the core of cluster model 1 with the highest $\dot{T}$ and highest degree of nonstationarity in the regular field (for the model considered, $\dot{T} \simeq(1.6 \pm 0.2) T / \tau_{\text {v.r. }}$, which is $8.1 \pm 1.5$ times greater than the rate of core heating due to stellar encounters). Danilov and Putov [7] determined the correlation functions using the results of numerical simulations of dynamical OCl models [11].

Theoretical study of the turbulence of stellar motions in $\mathrm{OCl}$ (and in their models) is of obvious interest. However, before undertaking such studies one has to analyze the entire spectrum of fluctuations of such systems and the instability of these fluctuations, reveal all (or most of) the instabilities of fluctuations in OCl models (for fluctuations in plasma, see [12, 13]; according to Volkov et al. [12], the main criterion of plasma turbulence is the broadening of the fluctuation spectrum, chaotization of fluctuation amplitudes and phases, and energy flow across the spectrum). Currently, such detailed data are unavailable for the dynamics of $\mathrm{OCl}$ models. Note that the authors of theoretical studies [3, 14-17] and other investigations use the differential equations for single-particle distribution functions and two-particle correlation functions to analyze spatially nonuniform stellar systems. Such equations are usually written with various simplifying assumptions. So far, our study [7] has been the only one to involve the computation of correlation functions based on the results of numerical simulations of dynamical $\mathrm{OCl}$ models. We believe that the lack of such studies is due to the exponential instability of stellar trajectories in such systems $[18,19]$ and the low accuracy of integration of the equations of stellar motion over sufficiently long time intervals [20]. Integration is usually performed using difference schemes of order no greater than four (see [18-20]).

Danilov and Dorogavtseva [11] computed the phase space coordinates (PSCS) of stars in $\mathrm{OCl}$ models by integrating the equations of stellar motion over a time interval $t \in\left[0, t_{\mathrm{m}}\right]$, where $t_{\mathrm{m}} \simeq 5.1 \tau_{\text {v.r. }}$, using 10th and 11 th order difference schemes. Let $t_{0}$ be the time interval of the dynamic evolution of an $\mathrm{OCl}$ model during which the system meets the statistical criterion of the accuracy of the computation of PSD [21]. In the vicinity of the centers of $\mathrm{OCl}$ models [11] $t_{0} / \tau_{\text {v.r. }} \simeq 3.0-3.9$, and $t_{0} / \tau_{\text {v.r. }} \simeq 3.6-5.1$ at the periphery of the models. In the models [11], the maximum fractional error of the computed cluster energy over the time interval $t_{\mathrm{m}}$ is equal to $(1-4) \times 10^{-13}$, and the accuracy of the computed phase-space density function (PSDF) of the cluster over the time interval $t_{0}$ can be considered to be sufficiently high to allow conclusions about the statistical properties of the PSDF to be made. In the $t \in\left[0, t_{0}\right]$ time interval the accuracy of the correlation distributions determined by Danilov and Putkov [7] proves to be sufficient to allow conclusions about the properties of these distributions. According to Danilov and Putkov [7], a comparison of the studied distributions or dependences (e.g., fluctuation spectra or dispersion curves) obtained using 10th and 11 th order integration methods provides yet another efficient tool to control the computation accuracy (see below).

Of interest are estimates of the PSD correlation time $\tau_{c}=\tau_{c}^{(f)}$ for OCl models [11]. No such estimates are reported in [7], because the above study did not involve the computation of two-time correlations of PSD $f$. The correlation times $\tau_{c}^{(f)}$ can be used to compute the average phase velocity $v_{f}$ of PSD fluctuations in $\mathrm{OCl}$ models (see, e.g., [12, 13]). It is also of interest to determine the average phase velocities $v_{r}, v_{v}$ of fluctuations in the $r$ and $v$ spaces for $\mathrm{OCl}$ models from the $\tau_{c}$ and $r_{c}$ presented in [7]. So far, no such estimates have been reported for OCl models.

After Miller's paper [22], a number of authors analyzed the motion instability in a many-body problem (see, e.g., [23-25]). In the case of systems that are close to stable dynamic equilibrium [18] the exponential instability of stellar trajectories results in the growth of the entropy of the system [26], p. 105; [27], p. 506.

Kandrup [23-25], Gurzadyan and Savvidy [28], Bocaletti et al. [29], and Danilov and Leskov [30] used different methods to study the separation of close trajectories in nonstationary stellar systems and the influence of collective effects on stellar trajectories. Kandrup [23-25] and Bocaletti et al. [29] estimated the time $\tau_{\text {v.r. }} \sim \bar{t}_{\text {cr }}$ for elliptical galaxies in terms of the "geometric" approach. Bocaletti et al. [29] obtained such estimates using the virial theorem assuming that the systems in question are close to stable equilibrium. Numerical simulations by Kandrup and Smith [31, 32] and other authors confirmed the theoretical estimates of $\tau_{\mathrm{v} . r .} \sim \bar{t}_{\mathrm{cr}}$ obtained by Kandrup [23-25] and Bocaletti et al. [29]. Danilov and Leskov [30] studied the maximum characteristic Lyapunov exponents $\lambda$ for stellar trajectories in $\mathrm{OCl}$ models with different degrees of non-stationarity, and showed that the exponents $\lambda$ averaged over all stellar trajectories decrease in passing from models with intermediate to higher degrees of non-stationarity. This pattern indicates the decrease of the degree of stochasticity in highly nonstationary systems.

Kandrup [33] theoretically discussed the dynamics of collisionless gravitating systems with PSD distribution $f_{0}$ in the vicinity of a time-dependent state of unstable equilibrium. Such systems should exhibit PSD fluctuations $\delta f$ about the distribution $f_{0}$ corresponding to the saddle point associated with a local 
Table 1. Correlation time $\tau_{c}^{(f)}$, initial time scale of violent relaxation $\tau_{\text {v.r. }}$, phase velocities of fluctuations, and the root mean square velocity $v_{c}$ of stellar motions in the cores of $\mathrm{OCl}$ models

\begin{tabular}{c|c|c|c|c|c|c}
\hline & 1 & 2 & 3 & 4 & 5 & 6 \\
\hline $\begin{array}{c}\tau_{c}^{(f)} / \tau_{\text {v.r. }} \\
\tau_{\text {v.r. }}[11], \\
M y r\end{array}$ & $0.98 \pm 0.14$ & $0.60 \pm 0.05$ & $0.52 \pm 0.03$ & $0.64 \pm 0.06$ & $0.14 \pm 0.02$ & $0.10 \pm 0.01$ \\
$\begin{array}{c}v_{f}, \\
\mathrm{pc} / \mathrm{Myr}\end{array}$ & $0.027 \pm 0.001$ & $0.051 \pm 0.004$ & $0.056 \pm 0.003$ & $0.053 \pm 0.005$ & $0.26 \pm 0.04$ & $0.27 \pm 0.03$ \\
$\begin{array}{c}v_{v}, \\
\mathrm{pc} / \mathrm{Myr}\end{array}$ & $0.012 \pm 0.001$ & $0.013 \pm 0.001$ & $0.021 \pm 0.005$ & $0.063 \pm 0.007$ & $0.17 \pm 0.01$ & $0.26 \pm 0.02$ \\
$\begin{array}{c}v_{r}, \\
\mathrm{pc} / \mathrm{Myr}\end{array}$ & $0.007 \pm 0.001$ & $0.008 \pm 0.001$ & $0.001 \pm 0.0001$ & $0.011 \pm 0.002$ & $0.009 \pm 0.002$ & $0.010 \pm 0.001$ \\
$\begin{array}{c}v_{c}, \\
\mathrm{pc} / \mathrm{Myr}\end{array}$ & $0.56 \pm 0.02$ & $0.56 \pm 0.01$ & $0.54 \pm 0.01$ & $0.57 \pm 0.01$ & $0.58 \pm 0.01$ & $0.55 \pm 0.01$ \\
$v_{c} / v_{f}$ & $20.7 \pm 1.0$ & $10.9 \pm 0.9$ & $9.7 \pm 0.6$ & $10.8 \pm 1.0$ & $2.2 \pm 0.3$ & $2.0 \pm 0.2$ \\
\hline
\end{tabular}

extremum of the energy $H\left(f_{0}\right)$ of the average field of the system: in this case $H(f)-H\left(f_{0}\right)$ increases for some $\delta f$ and decreases for other $\delta f$ (see the analysis of formula (18) from [33]).

In the $\mathrm{OCl}$ models with different degrees of closeness to unstable equilibrium [34] the correlations in the phase-space positions of stars may increase with time, and in these cases the entropy decreases [7]. Because of the low density of $\mathrm{OCl}$ models and the influence of the external Galactic gravitational field, they are populated or even dominated by numerous poorly studied instabilities of collective stellar motions (see below). The only such instability theoretically studied so far is the one associated with homologous fluctuations of $\mathrm{OCl}$ cores [35]. In the case of such fluctuations the mass density $\rho$ in a spherical stellar system can be represented as $\rho(r, t)=\rho_{1}(r) \rho_{2}(t)$. An analysis of the instabilities of fluctuations of OCls and their models will make it possible to identify the development mechanisms and classify these instabilities (Jeans, Non-Jeans, beam, gradient, and other instabilities [36], which in some cases are similar to plasma instabilities). Instabilities of collective stellar motions determine both the irregular structure of OCls (which is more irregular than that of globular clusters), and the dynamic evolution of OCls (development of nonstationarity in the regular field, development of turbulence in stellar motions as a result of the interaction of different fluctuation modes, formation and growth of polarization clouds in $\mathrm{OCl}$ models, etc.). In view of this, it is of interest to study PSD fluctuations in OCls and analyze the instability of these fluctuations.
According to Volkov et al. [12, 13], the mutual correlation functions of the fluctuations of a number of plasma properties at two points at a distance $r$ apart can be used to construct the dispersion curves and analyze the instabilities of fluctuations in plasma. It is of interest to use this method to construct the frequency spectra (power spectra) and study a number of instabilities of PSD fluctuations in $\mathrm{OCl}$ models. So far, no such analyzes of $\mathrm{OCl}$ models have been performed. The results of such a study can be used both to discuss the dynamics of $\mathrm{OCl}$ models and to theoretically describe the dynamical processes in $\mathrm{OCl}$.

The aim of this study is:

1) to compute and discuss $\tau_{c}^{(f)}, v_{f}, v_{r}$, and $v_{v}$ for OCl models [11];

2) to compute the two-time and mutual correlation functions for PSD fluctuations in OCl models;

3) to compute the frequency spectra and dispersion curves for PSD fluctuations in $\mathrm{OCl}$ models;

4) to use the obtained results to analyze the dynamics of $\mathrm{OCl}$.

\section{ESTIMATES OF $\tau_{c}^{(f)}, v_{f}, v_{r}, v_{v}$ IN OCl MODELS}

Danilov and Dorogavtseva [11] studied models of a cluster consisting of $N=500$ solar-mass stars moving in a circular orbit of radius $R_{\mathrm{G}}=8200 \mathrm{pc}$ in the Galactic plane about the Galactic center (using the Galactic potential model [37]). The initial parameters of these numerical dynamical $\mathrm{OCl}$ models are listed in 


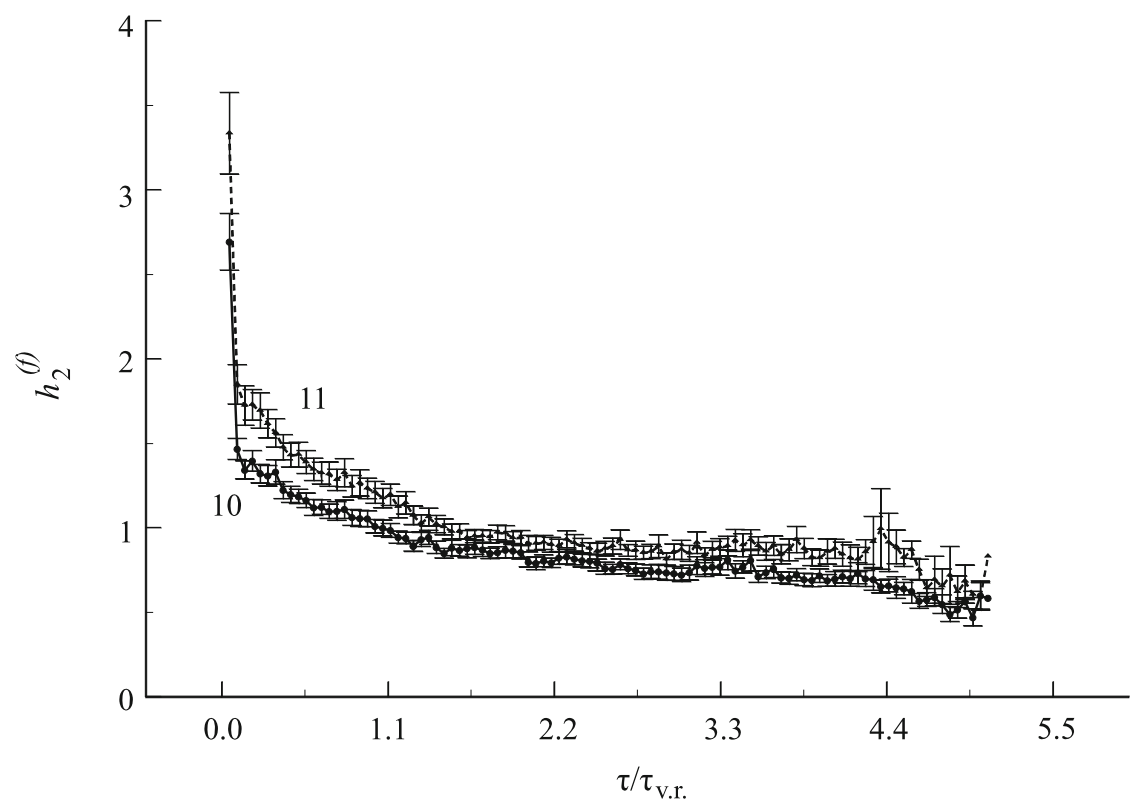

Fig. 1. Dependence of $h_{2}^{(f)}$ on $\tau$ for OCl model 1 at $\tau>0$. The numbers 10 and 11 indicate the order of the integration schemes employed to determine the PSCS used to compute these curves.

Table 1 in [38]; star cluster models $1-6$ in [11,38] are numbered in the order of decreasing degree of nonstationarity in the regular field. Each model initially consists of two spherical systems of uniform density (core and halo) with coincident centers of mass.

Following Klimontovich [39] (p. 136), and the notation adopted in [7], let us consider the following two-time correlation function to determine the correlation time $\tau_{c}^{(f)}$ in the space of $f$ values:

$$
g_{2}^{(f)}\left(t, t^{\prime}\right)=\overline{f(t) f\left(t^{\prime}\right)}-\overline{f(t)} \times \overline{f\left(t^{\prime}\right)},
$$

where the overline denotes averaging over all cluster stars; $f(t)$ and $f\left(t^{\prime}\right)$ are the values of PSD $f$ in the vicinity of the same star at times $t$ and $t^{\prime}$. Here we drop the other arguments $(\mathbf{r}, \mathbf{v})$ of the functions $f$ and $g_{2}^{(f)}$ for the sake of brevity. Like in our earlier paper [7], we estimate the PSD $f$ in the neighborhood of each star with the allowance for the phase-space coordinates of this star and of its five closest neighbors. Let $t^{\prime}=t+\tau$ and $\tau>0$. We now insert $t^{\prime}$ into (1) and average the function $g_{2}^{(f)}(t, t+\tau)$ over the time instants $t \in\left[0, t_{\mathrm{m}}-\tau\right]$ to determine the function $h_{2}^{(f)}(\tau)$ and its errors (at $\tau<0$ function $g_{2}^{(f)}(t, t+\tau)$ has to be averaged over time instants $\left.t \in\left[-\tau, t_{\mathrm{m}}\right]\right)$. Figure 1 shows the plot of function $h_{2}^{(f)}(\tau)$ for cluster model 1 at $\tau>0$. We do not plot the part where $\tau<0$, because the function is even: $h_{2}^{(f)}(\tau)=h_{2}^{(f)}(-\tau)$; see also Bendat and Piersol [40], p. 58.
Like in our previous paper [7], we define the correlation time $\tau_{c}^{(f)}$ to be equal to the time $\tau$ when $h_{2}^{(f)}(\tau)=h_{2}^{(f)}(0) / e$, where $e$ is the base of the natural logarithm. In this case we find for cluster model 1 $\tau_{c, 11}^{(f)}=(0.98 \pm 0.14) \tau_{\text {v.r. }}$ and $\tau_{c, 10}^{(f)}=(1.09 \pm 0.16) \tau_{\text {v.r. }}$. using the PSCS determined with the 11th and 10th-order integration schemes, respectively. The quoted error of $\tau_{c}^{(f)}$ is determined by the error of the computation of function $h_{2}^{(f)}(\tau)$ at $\tau=\tau_{c}^{(f)}$. The estimates $\tau_{c, 11}^{(f)}$ and $\tau_{c, 10}^{(f)}$ do not differ statistically from each other. Table 1 lists the estimates of $\tau_{c}^{(f)}$ for $\mathrm{OCl}$ models 1-6 obtained using the PSCS determined by the 11 th order integration method. According to Table 1 of this paper and Table 1 of [11], $\tau_{c}^{(f)} / \tau_{\text {v.r. }}$ and $\tau_{c}^{(f)}$ decrease with increasing model number and with decreasing degree of non-stationarity of the cluster model. Thus the correlations in the space of $f$ values decay most rapidly in the process of the evolution of model 6 . Given the correlation times in the $r$ space $\left(\tau_{c}^{(r)}\right)$ reported in [7], we find: $\tau_{c}^{(f)}<\tau_{c}^{(r)}$ for $\mathrm{OCl}$ models $1-6$. The ratio $\tau_{c}^{(r)} / \tau_{c}^{(f)}=q$ increases from $q \simeq 2.1$ in model 1 to $q \simeq 17.0$ in model 6 .

According to Volkov et al. [12] (p. 27), the average estimate of the phase velocity of PSD fluctuations can be determined from the relation between the correlation time and correlation radius, $v_{f} \simeq r_{c}^{(f)} / \tau_{c}^{(f)}$ (the average phase velocities $v_{r}$ and $v_{v}$ in the spaces 
of $r$ and $v$ can be determined in a similar way). Table 1 also lists the phase velocities $v_{f}, v_{v}$, and $v_{r}$ (in $\mathrm{pc} / \mathrm{Myr} ; 1 \mathrm{pc} / \mathrm{Myr} \simeq 1 \mathrm{~km} / \mathrm{s}$ ) determined using the PSCS computed with the 11th-order integration method and with the allowance for the correlation parameters in models $1-6$ adopted from [7]. It is evident from Table 1 that the phase velocities $v_{f}$ and $v_{v}$ in cluster models $1-4$ are substantially (by a factor of 5-20) smaller than the corresponding phase velocities for models 5 and 6 . In the case of $v_{r}$, the difference between the models is not so pronounced. The differences between the phase velocities $v_{f}$ and $v_{v}$ in models $1-4$ and the corresponding phase velocities in models 5 and 6 are possibly due to the longer wavelengths $\lambda$ of fluctuations of $f$ and $v$ values in models 5,6 . Let us now consider a radially propagating wave in the cluster model: $\delta f \propto \exp (-i \varphi)$, where $\varphi=\omega t-k_{r} r$, and $\omega$ and $k_{r}$ are the frequency of PSD fluctuations and the corresponding wavenumber, respectively. We then set $\varphi=$ const, $\omega=2 \pi / P=$ const, and $k_{r}=2 \pi / \lambda=$ const, and differentiate $\varphi$ with respect to time $t$ to obtain $v_{f}=\frac{d r}{d t}=\omega / k=\lambda / P$, where $P$ is the period of fluctuations. The periods of radial fluctuations of models $1-6$ differ little from each other (see, e.g., Table 2 in [30]). Therefore the differences of the wavelengths $\lambda$ of fluctuations of $f$ and $v$ in models $1-4$ and 5, 6 may be one of the factors causing the differences between $v_{f}$ and $v_{v}$ in these models. The high phase velocities $v_{f}$ and $v_{v}$ in models 5,6 may be due to the more extended and massive cores of these models compared to those of models $1-4$. Furthermore, during the periods of strongest compression toward the Galactic plane model 6 develops a toroidal structure with the equatorial plane close to the mid-plane of the Galaxy and with increased stellar number density inside the torus. This model also exhibits compression waves that propagate across the cluster core away from the $\zeta$ axis, passing through the center of mass of the model and perpendicular to the Galactic plane, at a velocity comparable to the average velocity of peculiar motion of the stars [30]. Note that the low phase velocities $v_{f}$ and $v_{v}$ in models 1-4 may be a result of the formation and superposition of several counterpropagating radial waves in these models (in this case the average phase velocity of the radial waves in the cluster may be small). Danilov and Ryazanov [41, 42] numerically integrated the Vlasov equations to find and study counterpropagating radial star flows that form in a nonstationary isolated spherical system with a constant PSD (a "water bag" type model). Such flows form in the core of the system during the periods of its compression and are due to the periodic motion of the region of zero PSD inside the phase-space domain occupied by the system. In the process, a density perturbation (a low-density region) develops periodically near the center of the system and is then carried by the flow of stars to the boundary of the cluster core. It is possible that such star flows develop more actively in the $\mathrm{OCl}$ models $1-4$ with a higher degree of non-stationarity compared to models 5, 6, and this circumstance affects the estimates of the correlation parameters $r_{c}^{(f)}$ and $\tau_{c}^{(f)}$, as well as those of $v_{f}$ for models $1-4$.

Table 1 lists the root mean square velocities $v_{c}$ (in $\mathrm{pc} / \mathrm{Myr}$ ) of the core stars with clustercentric distances $r \leq 0.5 R_{t}$ adopted from our previous paper [7]. According to this table, the velocities $v_{c}$ are 10-20 times greater than the phase velocities $v_{f}$ for models $1-4$ and about two times greater than $v_{f}$ for models 5,6 . Thus the average phase velocities of the fluctuations of $f, v, r$ in $\mathrm{OCl}$ models $1-6$ are sufficiently small compared to the root mean squared velocities $v_{c}$ of the stars in the core.

\section{MUTUAL CORRELATION FUNCTION}

Let $X(t, \mathbf{r})=f(t, \mathbf{r})-\overline{f(t, \mathbf{r})}$, where $f$ is determined by $n_{\text {st }}$ stars in the neighborhood of the point with coordinates $\mathbf{r}$ in the cluster model, and the overline denotes averaging over time interval $t \in\left[0, t_{\mathrm{m}}\right]$ (the origin of the $\mathbf{r}$ coordinate system coincides with the center of mass of the cluster and $t_{\mathrm{m}}$ is the maximum value of $t$ used to compute the correlations). For the sake of brevity, here we drop the argument $\mathbf{v}$ in functions $f$ and $X$. The quantity $X$ is usually referred to as the deviation or fluctuation (see $[12,13]$ ) and is assumed to be a random variable that forms a stationary random process. Following $[12,13]$, we write the mutual correlation function

$$
C_{x x}(\tau, \mathbf{r})=\frac{1}{t_{\mathrm{m}}} \int_{0}^{t_{\mathrm{m}}} X(t, \mathbf{r}) X(t+\tau, \mathbf{r}) d t,
$$

and the mutual correlation function of PSD fluctuations

$$
C_{x x}(\tau, \mathbf{r}, \Delta \mathbf{r})=\frac{1}{t_{\mathrm{m}}} \int_{0}^{t_{\mathrm{m}}} X(t, \mathbf{r}) X(t+\tau, \mathbf{r}+\Delta \mathbf{r}) d t .
$$

In the general case these functions can be written in the form of limits over $t_{\mathrm{m}}$ at $t_{\mathrm{m}} \rightarrow \infty$ of the right-hand sides of formulas (2) and (3). Here, like in the previous section of this paper, $\tau$ is the time delay.

Let $\mathbf{r}=\mathbf{0},|\Delta \mathbf{r}|=r$. In this case, the clustercentric distances of two points 1 and 2 are equal to 0 and $r$, respectively. Following [13], we denote the functions (2) determined by $n_{\text {st }}$ closest stars to each 

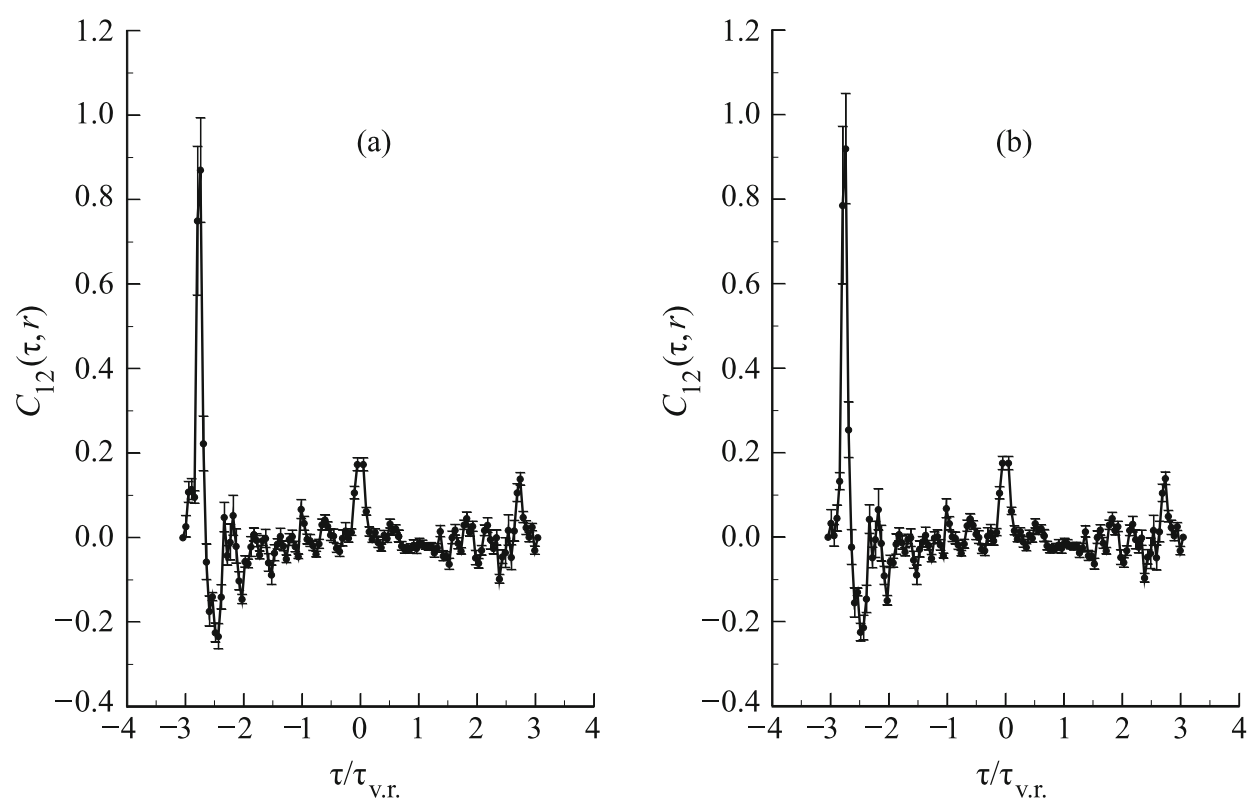

Fig. 2. Mutual correlation function in OCl model 1 for $r=1 \mathrm{pc}, n_{\mathrm{st}}=30$, and $n_{\mathrm{p}}=27$. Panels (a) and (b) show the plots computed using the PSCS determined by the 11 th and 10th order integration methods, respectively.

of the points 1 and 2 as $C_{11}(\tau)$ and $C_{22}(\tau)$, respectively. In this case we denote function (3) as $C_{12}(\tau, r)$. We now follow the technique proposed in [13] to consider the fluctuation $X$ as a wave packet propagating in a radial direction relative to the cluster center:

$$
X(t, r)=\int_{0}^{\infty} A(\omega) \exp (i(r k(\omega)-\omega t)) d \omega .
$$

Here the amplitude $A(\omega)$ and wavenumber $k(\omega)$ of the waves that constitute the packet depend on the frequency $\omega$ of these waves. In the case (4) the Fourier transform of function $C_{11}(\tau)$ (or $C_{22}(\tau)$ ) is equal to function $F(\omega)$, and the Fourier transform of function $C_{12}(\tau, r)$ is equal to:

$$
F_{1}(\omega)=F(\omega) \exp (\operatorname{irk}(\omega)),
$$

(see also formulas (3.41) or (4.8)-(4.11) in the book by Bendat and Piersol [40]). Thus an analysis of the Fourier transform of function $C_{12}(\tau, r)$ can be used to determine $\left|F_{1}(\omega)\right|=F(\omega)$ (the frequency spectrum) and $\frac{1}{r} \operatorname{Arg}\left(F_{1}(\omega)\right)=k(\omega)$ (the dispersion relation). Here $r k(\omega) \in[-\pi / 2, \pi / 2]$ is the phase of fluctuations of PSD $f$ with the frequency of $\omega$.

In this paper we consider a number of positions of point 2 on the sphere of radius $r$ centered at the center of mass of the cluster. To this end, we compute the coordinates of these points in the spherical coordinate system $(r, \theta, \phi)$ with a step of $\Delta \theta=\Delta \phi=\pi /(4 k)$ for $k=1,2,4$, and transform them to the right-hand Cartesian coordinate system $(\xi, \eta, \zeta)$ with the origin at the center of mass of the cluster (the $\zeta$ axis is directed perpendicularly to the mid-plane of the Galaxy; the $\xi$ axis lies in the midplane of the Galaxy, directed away from the Galactic center, and the $\eta$ axis points in the direction of the motion of the cluster in the Galactic plane). Together with point 1 , the total number of points $n_{\mathrm{p}}$ in whose neighborhood we consider the fluctuations $X$ is equal to $n_{\mathrm{p}}=27,115$, and 483 for $k=1,2$, and 4 , respectively.

Figure 2 shows the plots of the mean values of function $C_{12}(\tau, r)$ in model 1 for $r=1 \mathrm{pc}, n_{\mathrm{st}}=30$, and $n_{\mathrm{p}}=27$ in the case of $t_{\mathrm{m}}=t_{0}$, where $t_{0} \simeq 3 \tau_{\text {v.r. }}$. Here the mean $C_{12}(\tau, r)$ are averaged over $26 C_{12}(\tau, r)$ values corresponding to different positions of point 2 on the sphere of radius $r=1 \mathrm{pc}$. The vertical bars in Fig. 2 show the errors of the corresponding mean $C_{12}(\tau, r)$ values. Figures $2 \mathrm{a}$ and $2 \mathrm{~b}$ show the plots computed using the PSCS determined by the 11 th and 10th order integration methods, respectively. The differences between the $C_{12}(\tau, r)$ curves obtained using the 11th and 10th order integration methods in Fig. 2 are less noticeable than the corresponding differences between the plots of $h_{2}^{(f)}$ shown in Fig. 1 . The smaller difference between the curves in Fig. 2 is due to the use of smaller $t_{\mathrm{m}}$ compared to those used for Fig. 1 (the curves in Fig. 2 were computed using only the PSCS at $t \leq t_{0}$; the averaging of $f$ and $X(t, \mathbf{r}) X(t+\tau, \mathbf{r}+\Delta \mathbf{r})$ in (3) over $t$ was performed in the intervals: $t \in\left[0, t_{0}-\tau\right]$ at $\tau>0$ and $t \in\left[-\tau, t_{0}\right]$ at $\left.\tau<0\right)$. The differences between the curves $C_{12}(\tau, r)$ obtained using the 11 th and 10th or- 


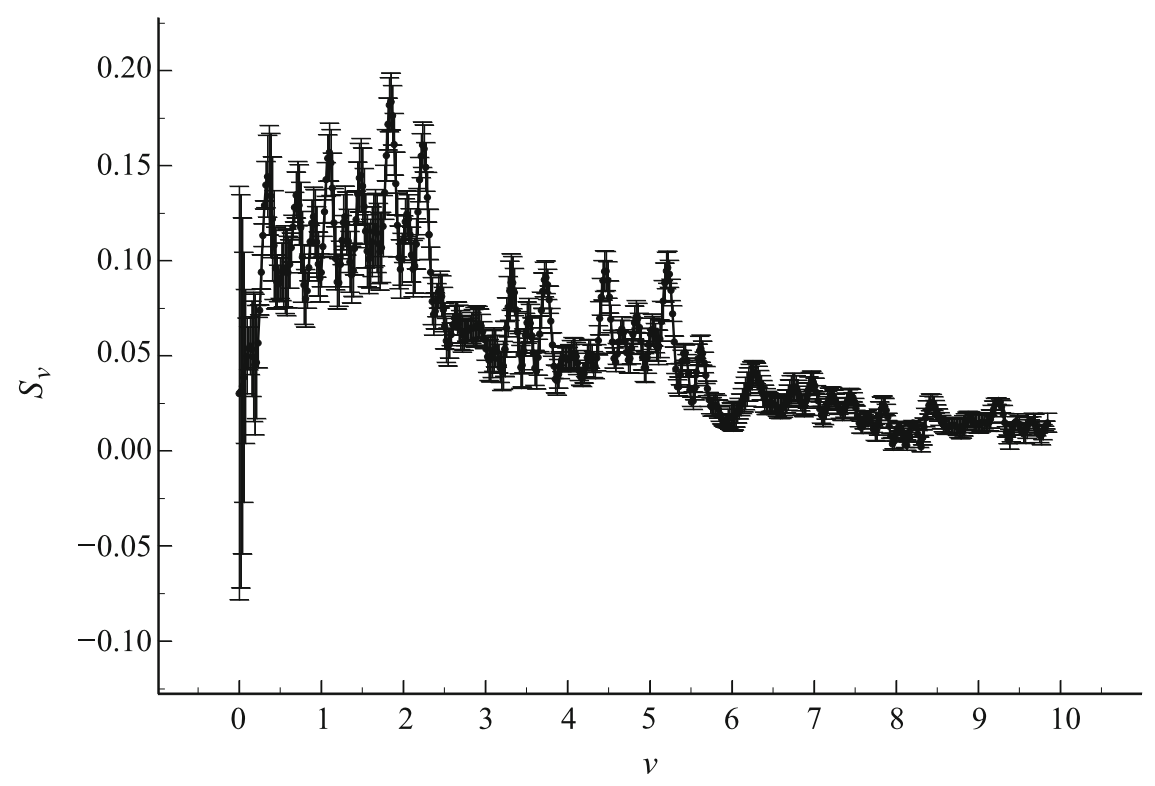

Fig. 3. Dependence of $S_{\nu}$ on $\nu$ for OCl model $1 ; r=1 \mathrm{pc}, n_{\mathrm{st}}=30$, and $n_{\mathrm{p}}=27$.

der integration methods are usually smaller or much smaller than the errors of $C_{12}(\tau, r)$ shown in Fig. 2, and these differences decrease with decreasing $|\tau|$. Note the significant asymmetry of function $C_{12}(\tau, r)$ relative to $\tau=0$ : the frequency spectrum is therefore not determined solely by the real part of the Fourier transform of function $C_{12}(\tau, r)$, see [40]. In Fig. 2 a number of local extrema of function $C_{12}(\tau, r)$ can be seen beyond the error bars of $C_{12}(\tau, r)$ at points $\tau_{j}=P_{j}$. Such extrema are indicative of the higher mutual correlations (of different signs) between the fluctuations of $f$ with periods $P_{j}$ in the vicinities of points 1 and 2 .

\section{FREQUENCY SPECTRA AND DISPERSION CURVES}

We use the program of fast Fourier transform (FFT) from the book by Gray [43] to compute the frequency spectra and dispersion curves of fluctuations of PSD $f$ in $\mathrm{OCl}$ models $1-6$. In our case cyclic frequencies $\nu=\omega /(2 \pi)$ are more convenient to use than circular frequencies $\omega$. In this case the period $P_{j}=1 / \nu_{r e, j}$ and the e-folding time scale $t_{j}=1 /\left(2 \pi \nu_{i m, j}\right)$ of the instability of PSD fluctuations can be determined from the complex conjugate pairs of the roots $\nu_{j}=\nu_{r e, j} \pm i \nu_{i m, j}$ of equation $k_{\nu}^{(0)}=0$. We separately computed the Fourier transforms $\mathcal{F}_{1}(\nu)$ and $\sigma_{\mathcal{F}}(\nu)$ of the mean $C_{12}(\tau, r)$ and their errors $\sigma_{C}(\tau, r)$. We then computed the relative phases (wavenumbers) of PSD fluctuations:

$$
\begin{aligned}
k_{\nu}^{(0)} & =\frac{1}{r} \operatorname{Arg}\left(\mathcal{F}_{1}(\nu)\right), \\
k_{\nu}^{(1,2)} & =\frac{1}{r} \operatorname{Arg}\left(\mathcal{F}_{1}(\nu) \pm \sigma_{\mathcal{F}}(\nu)\right) .
\end{aligned}
$$

We use the functions $k_{\nu}^{(0,1,2)}$ to estimate the frequencies $\nu$ and their errors.

Figure 3 shows a plot of function $S_{\nu}=\left|\mathcal{F}_{1}(\nu)\right| / \tau_{\text {v.r. }}$. for model 1 based on the mean values of $C_{12}(\tau, r)$ and the errors of these mean values. In this figure the $S_{\nu}$ values are in the units of $\mathrm{Myr}^{6} / \mathrm{pc}^{12}$; hereafter $\nu$ is equal to the number of cycles of PSD $f$ during time $\tau_{\text {v.r. }}$. The $S_{\nu}$ dependence shows a number of local maxima exceeding the errors of $S_{\nu}$ and associated with the local extrema of function $C_{12}(\tau, r)$. In the $\nu \in[0.5,2.4]$ interval (lowfrequency domain) the values of function $S_{\nu}$ are two to three times greater than in the $\nu \in[2.6,5.2]$ interval (intermediate frequencies). Function $S_{\nu}$ decreases at higher frequencies $\nu$. It is evident from Fig. 3 that the low frequencies $\nu \in[0.5,2.4]$ corresponding to the fluctuations of the core and the entire cluster as a whole are the most important contributors to the average power of the fluctuations of PSD $f$ (see, e.g., [12]). The frequencies of the local maxima of $S_{\nu}$ in the intermediate-frequency domain are approximately two (sometimes three) times higher than those of the corresponding local maxima of $S_{\nu}$ in the low-frequency domain. This is indicative of the onset of resonances between the PSD fluctuations with different frequencies $\nu$ in response to the formation of $S_{\nu}$ in model 1 (the transfer of energy of the PSD fluctuations from low to high frequencies, to the domain 
of low-amplitude fluctuations, is possibly of resonant nature). The points of local maxima of $S_{\nu}$ correspond both to the stable (e.g., $\nu \simeq 1.85,3.35$ in Figs. 3, 4) and unstable fluctuations of PSD $f(\nu \simeq 1.13,1.36$, 4.41, in Figs. 3, 4). The local maxima on the curve $S_{\nu}$ indicate the elevated power and intensity of excitation of PSD fluctuations with frequencies in the vicinity of the points of these local maxima in model 1 . The curves $S_{\nu}=S_{\nu}^{(10)}$ and $S_{\nu}=S_{\nu}^{(11)}$ determined at $t_{\mathrm{m}}=t_{0}$ for OCl models based on PSCS computed using the 10th and 11th order integration methods, respectively, are practically indistinguishable. The frequencies $\nu$ of the corresponding local maxima $S_{\nu}^{(10)}$ and $S_{\nu}^{(11)}$ coincide with each other and the mean $\left|S_{\nu}^{(10)}-S_{\nu}^{(11)}\right| / S_{\nu}^{(11)}$ values averaged over all $\nu$ are approximately 1.9 times smaller than the mean $\sigma\left(S_{\nu}\right) / S_{\nu}^{(11)}$ values averaged over all $\nu$, where $\sigma\left(S_{\nu}\right)$ are the errors of $S_{\nu}^{(11)}$ shown by the vertical error bars in Fig. 3. We may therefore consider the accuracy of the PSCS employed to be sufficient for constructing and analyzing the curves $S_{\nu}$.

Let $\bar{S}_{\nu}$ be the mean $S_{\nu}^{(11)}$ value averaged over the domain of low $\nu$ corresponding to the frequencies of the fluctuations of the cluster core, and $\Delta S_{\nu}$, the average difference between the maximum and minimum values of $S_{\nu}^{(11)}$ in this domain of frequencies $\nu$. The initial core-to-halo radius ratios are the same for cluster models 1,2 , and 4 ; the number of stars in the cores of these models is equal to $N_{1}=100$, and the density of these models increases from model 1 to model 4 (the initial number of stars is the same in these models, and the initial radii of models 1,2 , and 4 decrease with the increasing number of the model). In cluster models 2, 3, 5, and 6 the initial number of stars inside the core and the core size increase from model 2 to model $6\left(N_{1}=400\right)$, while the initial halo radius is the same for all these models (see, e.g., Table 1 in [38]). The inferred quantities $\bar{S}_{\nu}^{\prime}=\bar{S}_{\nu} \tau_{\text {v.r }}$ and $\Delta S_{\nu}^{\prime}=\Delta S_{\nu} \tau_{\text {v.r. }}$ (in $\mathrm{Myr}^{7} / \mathrm{pc}^{12}$ ) for models 1,2 , and 4 are equal to $\bar{S}_{\nu}^{\prime}=5.8 \pm 0.4$, $2.3 \pm 0.7,3.3 \pm 0.7$ and $\Delta S_{\nu}^{\prime}=2.9 \pm 0.3,2.0 \pm 0.6$, $2.3 \pm 0.6$, respectively. The $\bar{S}_{\nu}^{\prime}$ and $\Delta S_{\nu}^{\prime}$ quantities decrease by a factor of about 15 and 14 , respectively, with increasing core size and mass in models 2,3 , 5 , and 6 (in passing from model 2 to model 6 ). Thus a change of the initial cluster model density has little effect on $\bar{S}_{\nu}^{\prime}$ and $\Delta S_{\nu}^{\prime}$, and the increase of the core size and mass of the cluster model decreases substantially the quantities $\bar{S}_{\nu}^{\prime}$ and $\Delta S_{\nu}^{\prime}$. Let $\overline{\Delta \nu}_{i}$ be the mean distance between the points of neighboring local maxima of function $S_{\nu}^{(11)}$ in the domain of low frequencies $\nu$ corresponding to the highest $S_{\nu}^{(11)}$ and
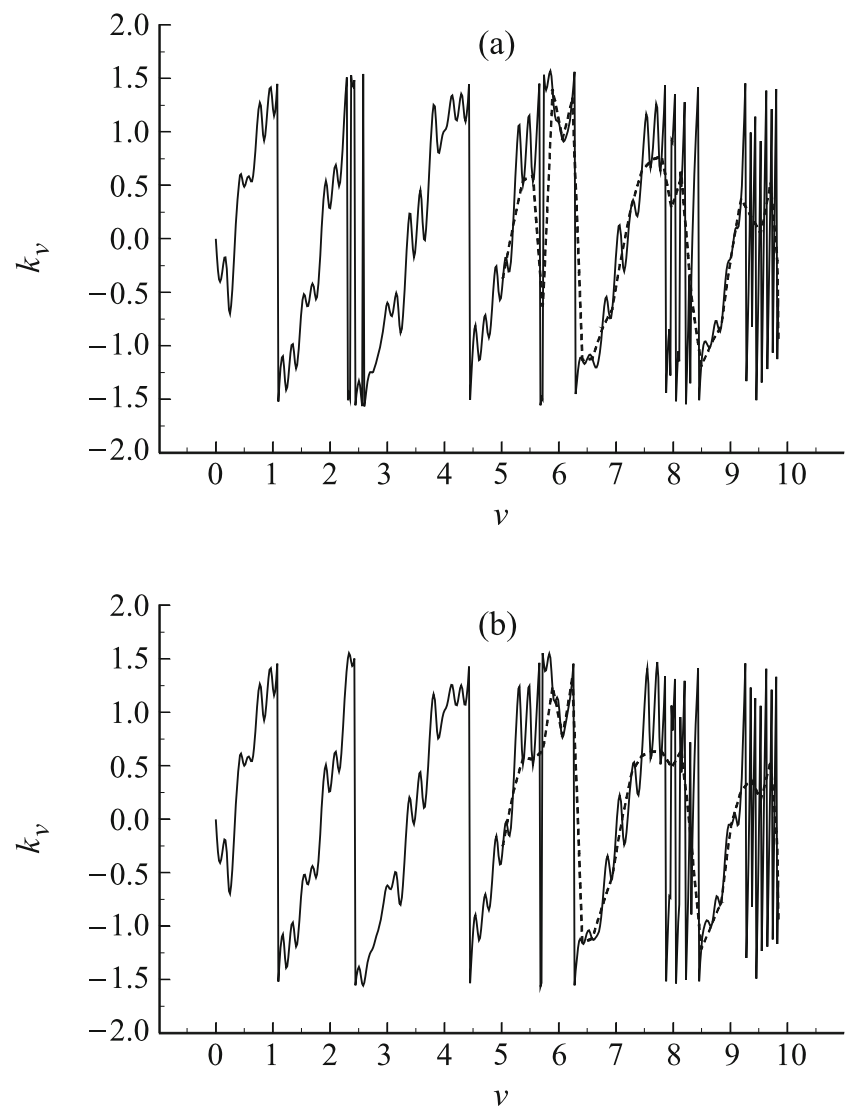

Fig. 4. Dispersion curves of PSD fluctuations for $\mathrm{OCl}$ model $1, r=1 \mathrm{pc}$, and $n_{\mathrm{st}}=30$. (a) The curve was computed with $n_{\mathrm{p}}=27$, (b) same with $n_{\mathrm{p}}=483$.

frequencies of the core fluctuations in cluster model $i$. The quantities $\overline{\Delta \nu}_{i}$ in models 1,2 , and 4 increase with increasing cluster density: $\overline{\Delta \nu}_{2} / \overline{\Delta \nu}_{1}=1.71 \pm 0.13$, $\overline{\Delta \nu}_{4} / \overline{\Delta \nu}_{1}=2.80 \pm 0.65$. The number of local maxima of functions $S_{\nu}^{(11)}$ in the low-frequency domains of the spectra with high $S_{\nu}^{(11)}$ decreases (by a factor of two) with increasing density of $\mathrm{OCl}$ models (in passing from model 1 to model 4 ). The greater degree of non-stationarity of model 1 compared to the other models must be due to the higher $\bar{S}_{\nu}^{\prime}$, greater number of local maxima of function $S_{\nu}^{(11)}$, and a higher density of these maxima in the low-frequency part of the spectrum.

Figure 4 shows the dependences of $k_{\nu}=k_{\nu}^{(0)}$ on $\nu$ for model 1 obtained for the function $C_{12}(\tau, r)$ plotted in Fig. 2a. We used the values $n_{\mathrm{p}}=27,483$. Varying $n_{\mathrm{st}}$ and $n_{\mathrm{p}}$ over a wide range allows the effect of random noise on the form of the dependence of $k_{\nu}$ on $\nu$ to be reduced in different intervals of frequencies $\nu$ (see, e.g., the almost vertical lines near $\nu \simeq 2.5$ in Figs. $4 \mathrm{a}$ and $4 \mathrm{~b}$ ). Note that the form and position 
of the "sinusoidal" portions of the $k_{\nu}$ dependences associated with the instabilities of PSD fluctuations remain practically unchanged. The farther such portions are from the $k_{\nu}=0$ line, the higher is the growth rate of such instabilities (in these cases the roots of the equation $k_{\nu}=0$ are complex conjugate). For example, two pairs of complex conjugate frequencies $\nu$ correspond to the "sinusoidal" portion of the curve $k_{\nu}$ in the vicinity of $\nu \simeq 1.1-1.5$ (fluctuation periods $P_{5}, P_{6}$ and the instability e-folding times $t_{5}, t_{6}$ for these roots are listed in Table 2; the pair of complex conjugate frequencies corresponding to $P_{6}$ and $t_{6}$ for model 1 was found in terms of a linear analysis of the instability of PSD fluctuations in the cluster core [35]). The intersection of the curve $k_{\nu}$ and the line $k_{\nu}=0$ corresponds to the real frequencies $\nu$ of PSD fluctuations (if it does not involve an abrupt change of phase $r k_{\nu}$ from $\mp \pi / 2$ to $\pm \pi / 2$; in this case the real part of the Fourier transform of function $C_{12}(\tau, r)$ attains the zero value in the neighborhood of the corresponding frequency $\nu$; such phase changes may be due to both the regular (non-random) variations of $C_{12}(\tau, r)$ with increasing $\nu$, and the random noise in the estimated $C_{12}(\tau, r)$ ). Table 2 lists the periods $P_{j}$ and e-folding times $t_{j}$ (in fractions of $\tau_{\text {v.r. }}$ ) for the PSD fluctuations with the highest instability growth increments in the intervals of frequencies $\nu$ considered for $\mathrm{OCl}$ models $1-6$. The $P_{j}$ and $t_{j}$ values listed in Table 2 of this paper were determined using the PSCS computed by the 11th order integration method. For model 1 we list the data for 17 pairs of such roots for $r=1 \mathrm{pc}$. The total number $n_{c}$ of root pairs in this model for $r=1 \mathrm{pc}$ reaches $41-42$. The value $n_{c}$ in model 1 varies with increasing $r$. We find it to be equal to $n_{c}=32-33, n_{c}=42, n_{c}=32$, and $n_{c}=22$ for $r=2,3,5$, and $9 \mathrm{pc}$, respectively. To determine the $P_{j}$ and $t_{j}$ values listed for models $1-6$ in Table 2, we fitted third-to-seventh order polynomials to the portion of the dispersion curve corresponding to the given root $\nu_{j}$; we then numerically found the roots of the resulting polynomial, and used the complex conjugate roots to compute $P_{j}$ and $t_{j}$.

The degree of instability of $\mathrm{OCl}$ models at different clustercentric distances may be related to the number of complex conjugate pairs of roots of equation $k_{\nu}=0$. In this case the resonances between the frequency of the orbital motion of the cluster in the Galaxy and the eigenfrequencies of the PSD in cluster models may play a certain part in the formation of instabilities at different clustercentric distances (see also [34]).

We use the following notation when writing down the values $P_{j}$ and $t_{j}$ in Table 2 . If the period $P_{j}$ determined from $k_{\nu}^{(0)}$ is at the boundary of the interval of the $P_{j}$ values inferred from $k_{\nu}^{(0,1,2)}$, we list it in Table 2 in parentheses with a prime on the side corresponding to the side of the interval where the period value is located. If the period $P_{j}$ determined from $k_{\nu}^{(0)}$ lies inside the interval of $P_{j}$ values determined from $k_{\nu}^{(1,2)}$, we list it in Table 2 as $P_{j} \pm \sigma\left(P_{j}\right)$, where $\sigma\left(P_{j}\right)$ is the standard deviation of $P_{j}$ determined from $k_{\nu}^{(1,2)}$. In the intervals of frequencies $\nu$ where the effect of random noise on $k_{\nu}^{(0)}$ due to random fluctuations of the mean $C_{12}(\tau, r)$ values cannot be suppressed by varying $n_{p}$ and $n_{s t}$, we use the method of locally weighted regression [44] to smooth the dependences $k_{\nu}$ to identify the signal at the noise level. The dashed lines in Fig. 4 show the $k_{\nu}$ curves for $\nu>5$ smoothed in such a way. In Table 2 the ":" sign indicates the periods $P_{j}$ and e-folding times $t_{j}$ determined using such smoothed $k_{\nu}$ curves.

The dispersion curves obtained at $t_{\mathrm{m}}=t_{0}$ for $\mathrm{OCl}$ models using the PSCS computed by the 10th and 11 th order integration methods are practically indistinguishable. The $P_{j}$ and $t_{j}$ values determined using the 10th and 11 th order dispersion curves $\left(P_{j}^{(10)}, t_{j}^{(10)}\right.$ and $P_{j}^{(11)}, t_{j}^{(11)}$ ) agree well with each other. To compare $P_{j}^{(10)}$ and $P_{j}^{(11)}$ and $t_{j}^{(10)}$ and $t_{j}^{(11)}$ for model 1, we selected several frequencies $\nu_{j}$ of unstable PSD fluctuations from different parts of the frequency interval considered in Fig. 4 (the adopted $j=5,6,10,14,16$ correspond to the $P_{j}$ and $t_{j}$ values for model 1 listed in Table 2). Let $\Delta P=\overline{\Delta P_{j}}$ and $\Delta t=\overline{\Delta t_{j}}$ be the mean $\Delta P_{j}=\left|P_{j}^{(11)}-P_{j}^{(10)}\right|$ and $\Delta t_{j}=\left|t_{j}^{(11)}-t_{j}^{(10)}\right|$ values averaged over the above $j$, and $\sigma_{P}$ and $\sigma_{t}$, the mean errors $\sigma\left(P_{j}\right)$ and $\sigma\left(t_{j}\right)$ of the $P_{j}$ and $t_{j}$ values from Table 2 averaged over the same $j$. We then have $\Delta P \simeq 0.68 \sigma_{P}, \Delta t \simeq 0.85 \sigma_{t}, \overline{\left(\Delta P_{j}\right) / P_{j}} \simeq 0.002$, and $\overline{\left(\Delta t_{j}\right) / t_{j}} \simeq 0.04$. Thus the 11 th and 10th order $P_{j}$ and $t_{j}$ estimates agree well with each other, and the accuracy of the PSCS employed can be considered to be sufficiently high to allow the construction and analysis of the dispersion curves.

According to Table 2, $P_{6}=(36.4-36.9)^{\prime} \mathrm{Myr}$ and $t_{6}=81.3 \pm 2.5 \mathrm{Myr}$ for model 1 (here we use $\tau_{\text {v.r. }}=49.9 \mathrm{Myr}$ in accordance with [11]). For this model the period $P_{\gamma}$ and the e-folding time $t_{\lambda}$ of the instability of PSD fluctuations determined in terms of a linear collisionless approximation by analyzing the PSD fluctuations associated with homologous fluctuations of the cluster core are equal to $P_{\gamma}=37.4 \pm 1.8 \mathrm{Myr}$ and $t_{\lambda}=53.7 \pm 2.9 \mathrm{Myr}$ (see columns 5 and 6 in Table 2 in [34]). Hence the estimates of $P_{6}$ and $P_{\gamma}$ agree well with each other. The e-folding time $t_{6}$ is about one and a half times longer than $t_{\lambda}$, which is most likely due to the nonlinear 

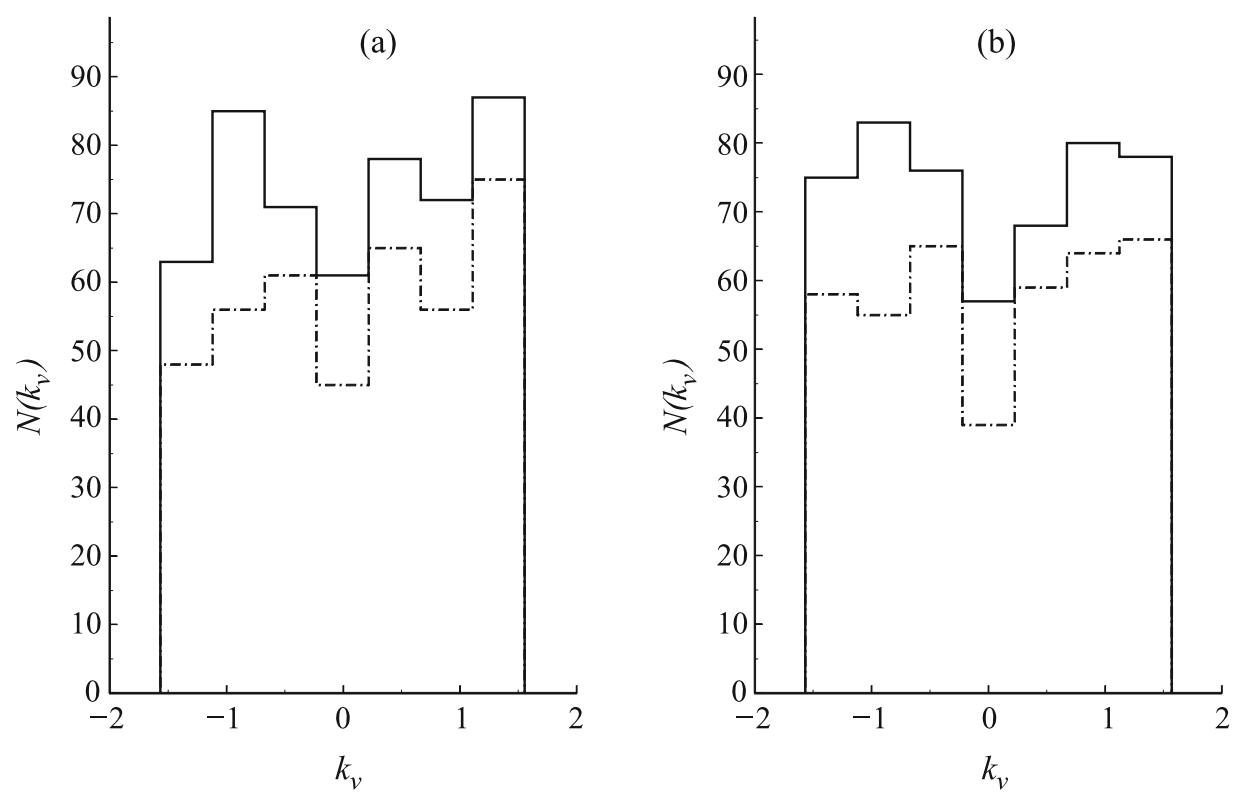

Fig. 5. Histograms of $N\left(k_{\nu}\right)$ for OCl model 1 for $r=1 \mathrm{pc}, n_{\mathrm{st}}=30$ : (a) $n_{\mathrm{p}}=27$ and (b) $n_{\mathrm{p}}=483$. The dashed-and-dotted lines show the histograms for frequencies $\nu \leq 7.72$.

nature of PSD fluctuations and the effect of stellar encounters on PSD fluctuations in the core of model 1 . The point is that the time intervals $\Delta t \simeq t_{\lambda}$ and $t_{6}$ are long enough for stellar encounters to somewhat "heat up" the instability development region, thereby reducing the rate of the instability development; the initial relaxation time due to stellar encounters is equal to $\tau_{\mathrm{st}}=121.9 \pm 12.2 \mathrm{Myr}$ and $\tau_{\mathrm{st}}=72.6 \pm 7.2 \mathrm{Myr}$ for models 1 and 6 , respectively [45] (Table 3), and therefore $\Delta t<\tau_{\text {st }}$ for model 1 . The asterisk subscript " *" in Table 2 is used to mark the $P_{j}, t_{j}$ pairs that are closest to the theoretical estimates $P_{\gamma}, t_{\lambda}$ for models 1-6 (see columns 4 and 5 in Table 2 in [34]). The mean asterisk ( "*") marked $P_{j}$ and $t_{j}$ values averaged over models $1-6$ are equal to $P_{j}=(1.09 \pm 0.06) P_{\gamma}$ and $t_{j}=(1.56 \pm 0.18) t_{\lambda}$, respectively.

According to Table 2, the periods of unstable fluctuations in model 1 range from $P_{1} \simeq 345 \mathrm{Myr}$ to $P_{17} \simeq 5.3 \mathrm{Myr}$. Hence $P_{1}>P_{\mathrm{G}} \simeq 222.1 \mathrm{Myr}$, where $P_{\mathrm{G}}$ is the orbital period of the cluster model about the Galactic center (see, e.g., Table 3 for models 1, 6 from [45]). The external gravitational field of the Galaxy appears to play an appreciable part in the development of fluctuations of model 1 with the periods of $P_{1}$ and $P_{2}$; the regular gravitational field of the cluster plays a major part in the development of large-scale fluctuations with the periods of $P_{3}-P_{6}$. Fluctuations with periods $P_{7}-P_{8}$ may be due to the nonhomologous nature of fluctuations of model 1 and its core (see [35] for estimates of the frequencies of homologous and nonhomologous fluctuations of elliptical models of cluster halos). Low-amplitude fluctuations with periods $P_{9}-P_{17}$ may be due to the formation of polarization clouds as a response of the system to the passage of a star, development of resonances between the fluctuations of $f$ at different frequencies (see above), or the turbulence in stellar motions in cluster model 1 ( see [7]).

Note that the phases $r k_{\nu}$ of unstable PSD fluctuations shown in Fig. 4 are distributed rather uniformly along the $k_{\nu}$ axis. Multiple reversals of the sign of $k_{\nu}$ in the frequency interval considered in Fig. 4 indicate the formation of a number of counterpropagating running radial waves of PSD fluctuations in model 1. Similar reversals of the sign of $k_{\nu}$ can also be seen on the dispersion curves of cluster models 2-6.

Figures $5 \mathrm{a}$ and $5 \mathrm{~b}$ show the histograms of the distributions $N\left(k_{\nu}\right)$ of $k_{\nu}$ used to construct the plots in Figs. $4 \mathrm{a}$ and $4 \mathrm{~b}$, respectively. The dotted lines in Fig. 5 show the histograms for frequencies $\nu \leq 7.72$ (this excludes the parts of the dispersion curves that are highly distorted by random noise in $k_{\nu}^{(0)}$ due to random variations of the mean $\left.C_{12}(\tau, r)\right)$. The histograms $N\left(k_{\nu}\right)$ for $\mathrm{OCl}$ models $1-6$ show a rather uniform distribution of the phases of PSD fluctuations, and this fact also indicates that a number of counterpropagating running radial waves of PSD fluctuations form in the models and account for the low average phase velocities of such waves.

The large number of unstable fluctuations and the high excitation intensity and power of such fluctuations in model 1 (see the text above and [12]) can be 


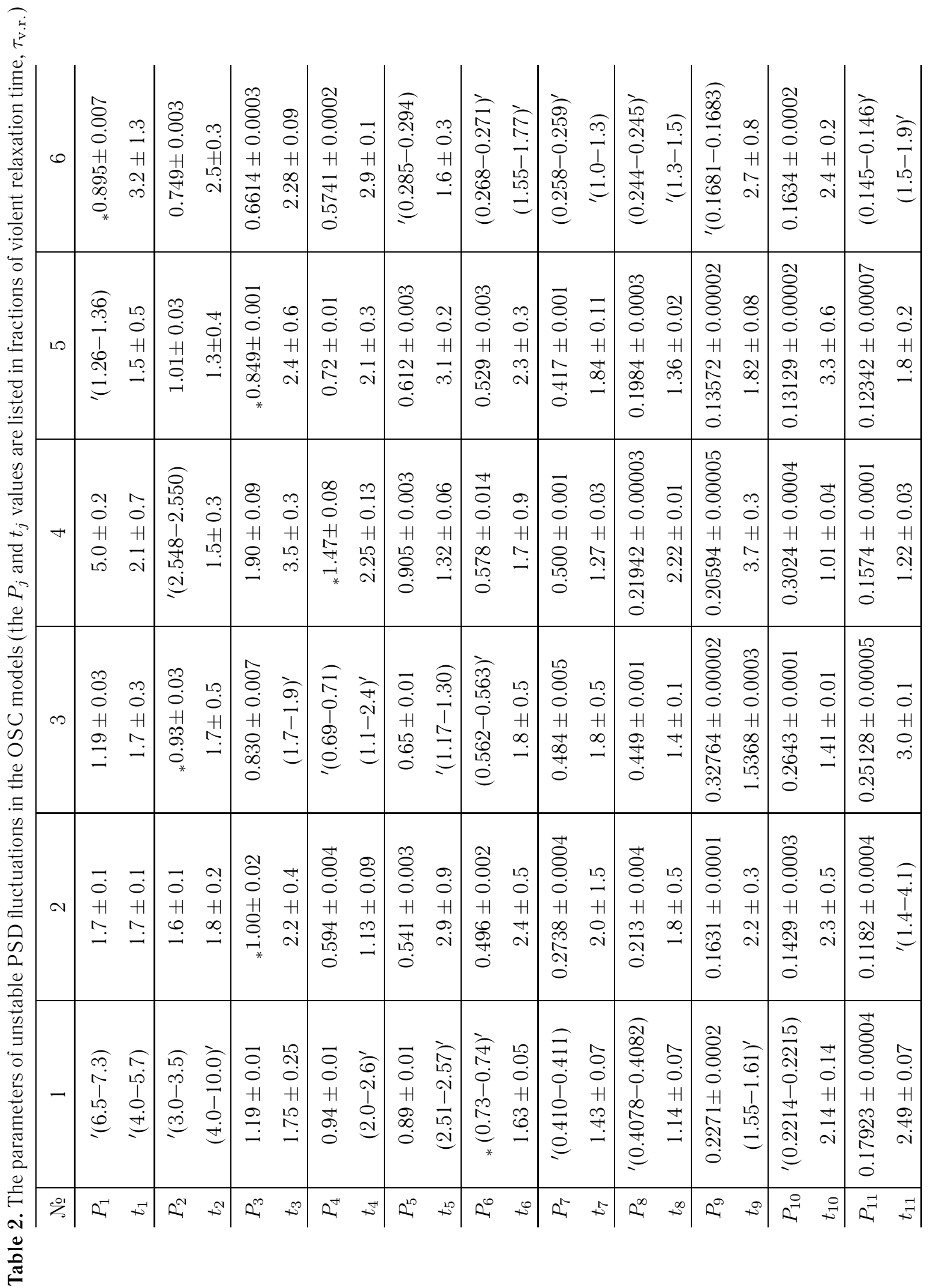




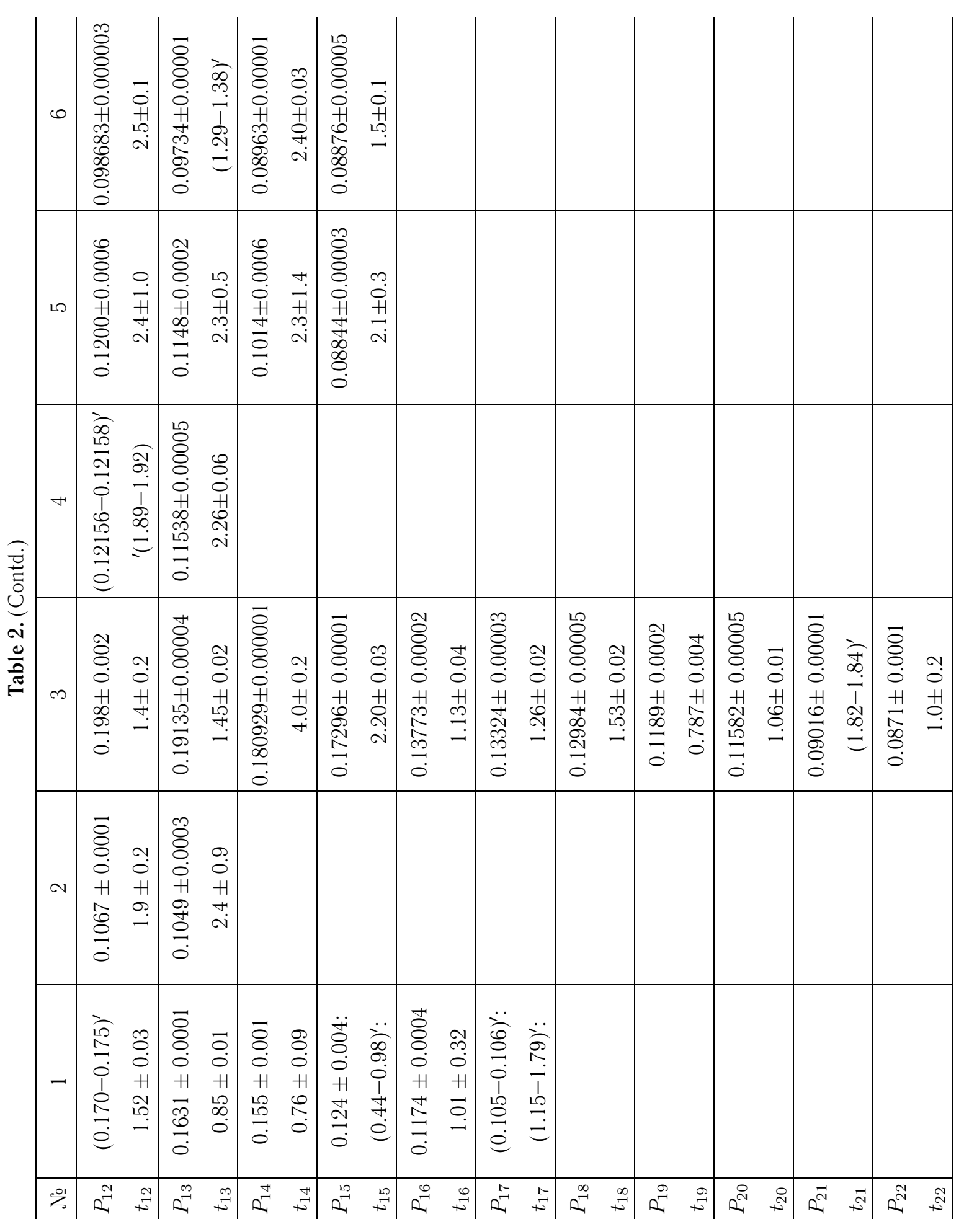


viewed as evidence for turbulence developing in the core of this cluster model.

Collective motions in the $\mathrm{OCl}$ and the instabilities of these motions determine the irregularity of the structure of such clusters. A number of structural $\mathrm{OCl}$ features (irregular core shapes inconsistent with the equilibrium state of the cluster, steplike features in the radial density profiles, etc. [46-49]) are known to be indicative of the non-stationarity of these clusters in the regular field. The instabilities of collective motions in these features may explain the structures and dynamical processes observed in $\mathrm{OCl}$ and their models (see [7, 34, 49] for a discussion of the dynamical evolution of such clusters).

The phenomena of incomplete violent relaxation and metastable equilibrium (QSS-quasi-stationary states) observed in the models of the systems with long-range interactions have been discussed extensively in the recent studies dedicated to such systems (see $[2,3,50,51])$. According to these studies, it is not yet entirely clear what causes the development of these states in such systems. In the case of $\mathrm{OCl}$ the development of metastable equilibrium states may be due, among other factors, to temporary suppression of the instability of radial fluctuations of the core of the system resulting from its "heating" by fluctuations of the regular field of the cluster. The ensuing temporary expansion of the cluster core and of the entire cluster (after the decay of collective fluctuations and the transformation of the thermal energy of collective fluctuations into the thermal energy of stellar motions) results in the cooling of the core and the development of gravitational instability over the time $t \leq \tau_{\text {st }}$, where $\tau_{\text {st }}$ is the relaxation time due to stellar encounters (see [34] for a discussion about the stages of temporary virialization, which last up to $10^{8} \mathrm{yr}$ in $\mathrm{OCl}$ models, and the subsequent development of the cluster core fluctuations). The existence and instability of such fluctuations in $\mathrm{OCl}$ models were studied theoretically by Danilov [34, 35], and confirmed in this paper using yet another method.

\section{CONCLUSIONS}

1. We estimated the correlation times $\tau_{c}^{(f)}$ in the space of PSD $(f)$ values in OCl models $1-6$. The correlations in the space of PSD values disrupt most rapidly in the process of the evolution of model 6 . A comparison of the correlation times $\tau_{c}^{(r)}$ in the $r$ space with the results obtained in our earlier paper [7] showed that $\tau_{c}^{(f)}<\tau_{c}^{(r)}$ for all six considered $\mathrm{OCl}$ models. The $\tau_{c}^{(r)} / \tau_{c}^{(f)}$ ratio increases from about 2.1 in model 1 to about 17.0 in model 6 .

2. We estimated the mean phase velocities $v_{f}, v_{r}$, $v_{v}$ of the fluctuations of $f, r$, and $v$ in OCl models $1-6$.
The phase velocities $v_{f}$ and $v_{v}$ in models $1-4$ are 5 to 20 times higher than the corresponding phase velocities in models 5 and 6 , possibly due to the longer wavelengths of fluctuations of $f$ and $v$ in models 5 and 6 . The small $v_{f}$ and $v_{v}$ values in models $1-4$ may be due to the formation and superposition of several counterpropagating running radial waves in these models. Phase velocities $v_{f}$ are 10-20 and about 2 times smaller than the mean squared velocities $v_{c}$ of stellar motions in the cores of models $1-4$ and 5,6 , respectively.

3. We computed for the first time the mutual correlation functions of $\mathrm{PSD}$ fluctuations of $\mathrm{OCl}$ models. We found a number of local extrema of function $C_{12}(\tau, r)$ that are beyond the $C_{12}(\tau, r)$ error limits at points $\tau_{j}=P_{j}$. These extrema are indicative of the elevated mutual correlations (of either sign) between the PSD fluctuations with periods $P_{j}$ at the cluster center and at the clustercentric distance $r$. We computed the frequency spectrum of the PSD fluctuations corresponding to function $C_{12}(\tau, r)$. Low frequencies $\nu \in[0.5,2.4]$ corresponding to the fluctuations of the core and the entire cluster as a whole are the major contributors to the average power of the PSD fluctuations. We found a number of local maxima in the frequency spectrum that are outside the errors of the computed spectrum, and these maxima are indicative of the elevated excitation power and intensity of PSD fluctuations with frequencies $\nu$ emerging from the neighborhood of the points of the local maxima of $S_{\nu}$. We point out that resonances may play a certain role in the formation of different parts of the frequency spectrum. A comparative analysis of the spectra of models $1-6$ suggests that the higher non-stationarity degree of model 1 compared to the other models is due to the greater $\bar{S}_{\nu}^{\prime}$ values, the greater number of local maxima of function $S_{\nu}$, and the higher density of these maxima in the low-frequency part of the spectrum.

4. We computed for the first time the dispersion curves for PSD fluctuations in numerical dynamical $\mathrm{OCl}$ models. We confirmed the presence of the known unstable PSD fluctuations in the cores of the cluster models that are associated with homologous fluctuations of the cores. We found several dozen new unstable PSD fluctuations in each of the models considered. The number of such fluctuations decreases nonmonotonically with clustercentric distance $r$. We discuss the possible development mechanisms of such fluctuations in $\mathrm{OCl}$ models. Unstable high-frequency PSD fluctuations may well be due to the development of polarization clouds resulting from the passage of stars through $\mathrm{OCl}$ models, the onset of resonances between the PSD fluctuations at different frequencies, or the turbulence in stellar motions in cluster model 1 . 
5. The phases $r k_{\nu}$ of unstable PSD fluctuations are distributed sufficiently uniformly along the $k_{\nu}$ axis. The histograms $N\left(k_{\nu}\right)$ also show a sufficiently uniform distribution of $k_{\nu}$ values. Multiple reversals of the sign of $k_{\nu}$ in the considered frequency interval on the dispersion curves show that counterpropagating running radial waves of fluctuations of $f$ form in cluster models $1-6$.

6. We consider the large number of unstable PSD fluctuations and the elevated power, and hence intensity, of excitation of such fluctuations in model 1 as evidence for the development of turbulence in cluster model 1 .

7. A detailed study of stellar velocities in OCls from the list [52] may provide further data (in addition to those already obtained by Danilov and Putkov [49], which are available at http://cdsarc.u-strasbg.fr/cgi-bin/ /Vizier?-source=J/AZh/89/674) on the non-stationarity of these clusters and the instability of PSD fluctuations marked by the "*" subscript in Table 2 . Such a study may use the radial variations of the stellar velocity dispersion in the cluster and the dispersions of tangential and radial velocities of the stars in the extended neighborhoods of these clusters (the increase of stellar velocity dispersion with clustercentric distance is an important kinematic indicator of $\mathrm{OCl}$ nonstationarity [34]).

\section{ACKNOWLEDGMENTS}

We are grateful to S. Yu. Gorda, senior research fellow of the Astronomical Observatory of the Ural Federal University, for useful discussions of the study. This work was supported in part by the Federal Targeted Programme "Research and Development in Priority Fields of Science and Technology Complex of Russia in 2007-2012" (state contract no. 16.518.11.7074).

\section{REFERENCES}

1. P. H. Chavanis, Physica A 361, 81 (2006).

2. P. H. Chavanis, Physica A 387, 787 (2008).

3. P. H. Chavanis, Physica A 387, 1504 (2008).

4. T. Baertschiger and F. S. Labini, Physical Rev. D. 69 , 123001 (2004).

5. M. Botaccio, M. Montuori, L. Pietronero, et. al., Mem. S. A. It. Suppl. 1, 120 (2003).

6. M. Botaccio, L. Pietronero, A. Amici, et al., Physica A 305, 247 (2002).

7. V. M. Danilov and S. I. Putkov, Astron. Rep. 56, 623 (2012).

8. V. M. Danilov, Astron. Rep. 46, 887 (2002).

9. S. J. Aarseth, Astronom. and Astrophys. 35, 237 (1974).

10. I. R. King, Astronom. J. 67, 471 (1962).
11. V. M. Danilov and L. V. Dorogavtseva, Astron. Rep. 52, 467 (2008).

12. Ya. F. Volkov, V. G. Dyatlov, and R. I. Mitina, Diagnostika turbulentnoi plazmy (Diagnostics of Turbulent Plasma) (Naukova Dumka, Kiev, 1983) [in Russian].

13. M. Bernard, G. Briffod, J. Bussac, et al., in Diagnostika plazmy (Atomizdat, Moscow, 1973), Iss. 3, p. 449.

14. G. Severne and M. J. Haggerty, Astrophys. and Space Sci. 45, 287 (1976).

15. I. H. Gilbert, Astrophys. J. 152, 1043 (1968).

16. I. H. Gilbert, Astrophys. J. 159, 239 (1970).

17. C. Nardini, S. Gupta, S. Ruffo, et. al., J. Stat. Mech. L01002 (2012).

18. J. Goodman, D. C. Heggie, and P. Hut, Astrophys. J. 415,715 (1993).

19. H. E. Kandrup, M. E. Magon, and H. C. Smith, Astrophys. J. 428, 458 (1994).

20. N. Komatsu, T. Kiwata, and S. Kimura, Physica A 387, 2267 (2008).

21. V. M. Danilov, Astron. Lett. 23, 322 (1997).

22. R. H. Miller, Astrophys. J. 140, 250 (1964).

23. H. E. Kandrup, Physics Lett. A 140, 97 (1989).

24. H. E. Kandrup, Physica A 73, 169 (1990).

25. H. E. Kandrup, Astrophys. J. 364, 420 (1990).

26. G. M. Zaslavskii and R. Z. Sagdeev, Vvedenie v nelineinuyu fiziku: ot mayatnika do turbulentnosti $i$ khaosa (Introduction into Nonlinear Physics: from the Pendulum to Turbulence and Chaos) (Nauka, Moscow, 1988) [in Russian].

27. Yu. L. Klimontovich, Statustucheskaya teoriya otkrytykh sistem (Statistical Theory of Open Systems) (TOO "Yanus," Mscow, 1995) [in Russian].

28. V. G. Gurzadyan and G. K. Savvidy, Astronom. and Astrophys. 160, 203 (1986).

29. D. Bocaletti, G. Pucacco, and R. Ruffini, Astronom. and Astrophys. 244, 48 (1991).

30. V. M. Danilov and E. V. Leskov, Astron. Rep. 49, 190 (2005).

31. H. E. Kandrup and H. Jr. Smith, Astrophys. J. 374, 255 (1991).

32. H. E. Kandrup and H. Jr. Smith, Astrophys. J. 399, 627 (1992).

33. H. E. Kandrup, Astrophys. J. 500, 120 (1998).

34. V. M. Danilov, Astron. Rep. 55, 473 (2011).

35. V. M. Danilov, Astron. Rep. 52, 888 (2008).

36. V. L. Polyachenko and A. M. Fridman, Ravnovesie i ustoichivost gravitiruyushchikh sistem (Equilibrium and Stability of Gravitating Systems (Nauka, Moscow, 1976) [in Russian].

37. S. A. Kutuzov and L. P. Osipkov, Sov. Astron. 24, 17 (1980).

38. V. M. Danilov and L. V. Dorogavtseva, Astron. Rep. 47, 483 (2003).

39. Yu. L. Klimontovich, Statisticheskaya fizika (Statistical Physics) (Nauka, Moscow, 1982) [in Russian]. 
40. J. S. Bendat and A. G. Piersol, Engineering Applications of Correlation and Spectral Analysis (Publ. J. Wiley and Sons, New York, 1980).

41. V. M. Danilov and A. P. Ryazanov, in Astronomogeodezicheskie issledovaniya, Ed. by K. A. Barkhatova (Ural State University, Sverdlovsk, 1985), p. 19.

42. V. M. Danilov and A. P. Ryazanov, Astron. Tsirkular 1487, 3 (1987).

43. D. F. Gray, The Observations and Analysis of Stellar Photospheres (Publ. J. Wiley and Sons, New York, 1976).

44. W. S. Cleveland and S. J Devlin, Journal of the American Statistical Association. 83, 596 (1988).
45. V. M. Danilov, Astron. Rep. 54, 514 (2010).

46. K. A. Barkhatova, Astronom. Zh. 33, 556 (1956).

47. A. F. Seleznev, Astron. Astrophys. Trans. 4, 167 (1994).

48. J. Binney and S. Tremaine, Galactic Dynamics, 6 ed. (Univ. Press, Princeton, 2008).

49. V. M. Danilov, S. I. Putkov, Astron. Rep. 56, 609 (2012).

50. P. H. Chavanis, Physica A 365, 102 (2006).

51. F. Bouchet, S. Gupta, and D. Mukamel, Physica A 389, 4389 (2010).

52. V. M. Danilov and A. F. Seleznev, Astron. Astrophys. Trans. 6, 85 (1994). 